Anticoagulants, Antiplatelets, and Thrombolytics 


\section{John M. Walker, SERIES EDITOR}

96. Hepatitis B and D Protocols: Volume 2, Immunology, Model Systems, and Clinical Studies, edited by Robert K. Hamatake and Johnson Y. N. Lau, 2004

95. Hepatitis B and D Protocols: Volume 1, Detection, Genotypes, and Characterization, edited by Robert K. Hamatake and Johnson Y. N. Lau, 2004

94. Molecular Diagnosis of Infectious Diseases, Second Edition, edited by Jochen Decker and Udo Reischl, 2004

93. Anticoagulants, Antiplatelets, and Thrombolytics, edited by Shaker A. Mousa, 2004

92. Molecular Diagnosis of Genetic Diseases, Second Edition, edited by Rob Elles and Roger Mountford, 2003

91. Pediatric Hematology: Methods and Protocols, edited by Nicholas J. Goulden and Colin G. Steward, 2003

90. Suicide Gene Therapy: Methods and Reviews, edited by Caroline J. Springer, 2003

89. The Blood-Brain Barrier: Biology and Research Protocols, edited by Sukriti Nag, 2003

88. Cancer Cell Culture: Methods and Protocols, edited by Simon P. Langdon, 2003

87. Vaccine Protocols, Second Edition, edited by Andrew Robinson, Michael J. Hudson, and Martin P. Cranage, 2003

86. Renal Disease: Techniques and Protocols, edited by Michael S. Goligorsky, 2003

85. Novel Anticancer Drug Protocols, edited by John K. Buolamwini and Alex A. Adjei, 2003

84. Opioid Research: Methods and Protocols, edited by Zhizhong Z. Pan, 2003

83. Diabetes Mellitus: Methods and Protocols, edited by Sabire Özcan, 2003

82. Hemoglobin Disorders: Molecular Methods and Protocols, edited by Ronald L. Nagel, 2003
81. Prostate Cancer Methods and Protocols, edited by Pamela J. Russell, Paul Jackson, and Elizabeth A. Kingsley, 2003

80. Bone Research Protocols, edited by Miep H. Helfrich and Stuart H. Ralston, 2003

79. Drugs of Abuse: Neurological Reviews and Protocols, edited by John Q. Wang, 2003

78. Wound Healing: Methods and Protocols, edited by Luisa A. DiPietro and Aime L. Burns, 2003

77. Psychiatric Genetics: Methods and Reviews, edited by Marion Leboyer and Frank Bellivier, 2003

76. Viral Vectors for Gene Therapy: Methods and Protocols, edited by Curtis A. Machida, 2003

75. Lung Cancer: Volume 2, Diagnostic and Therapeutic Methods and Reviews, edited by Barbara Driscoll, 2003

74. Lung Cancer: Volume 1, Molecular Pathology Methods and Reviews, edited by Barbara Driscoll, 2003

73. E. coli: Shiga Toxin Methods and Protocols, edited by Dana Philpott and Frank Ebel, 2003

72. Malaria Methods and Protocols, edited by Denise L. Doolan, 2002

71. Haemophilus influenzae Protocols, edited by Mark A. Herbert, Derek Hood, and E. Richard Moxon, 2002

70. Cystic Fibrosis Methods and Protocols, edited by William R. Skach, 2002

69. Gene Therapy Protocols, Second Edition, edited by Jeffrey R. Morgan, 2002

68. Molecular Analysis of Cancer, edited by Jacqueline Boultwood and Carrie Fidler, 2002

67. Meningococcal Disease: Methods and Protocols, edited by Andrew J. Pollard and Martin C. J. Maiden, 2001

66. Meningococcal Vaccines: Methods and Protocols, edited by Andrew J. Pollard and Martin C. J. Maiden, 2001 


\title{
Anticoagulants, Antiplatelets, and Thrombolytics
}

\author{
Edited by \\ Shaker A. Mousa \\ Albany College of Pharmacy, Albany, NY
}

Humana Press 来 Totowa, New Jersey 
(c) 2004 Humana Press Inc.

999 Riverview Drive, Suite 208

Totowa, New Jersey 07512

\section{www.humanapress.com}

All rights reserved.

No part of this book may be reproduced, stored in a retrieval system, or transmitted in any form or by any means, electronic, mechanical, photocopying, microfilming, recording, or otherwise without written permission from the Publisher. Methods in Molecular Medicine ${ }^{\mathrm{TM}}$ is a trademark of The Humana Press Inc.

All authored papers, comments, opinions, conclusions, or recommendations are those of the author(s), and do not necessarily reflect the views of the publisher.

This publication is printed on acid-free paper. $\infty$

ANSI Z39.48-1984 (American Standards Institute) Permanence of Paper for Printed Library Materials.

Production Editor: Mark J. Breaugh.

Cover design by Patricia F. Cleary.

Cover illustration: Potent inhibition of FGF2-induced angiogenesis by r-TFPI in the chick chorioallantoic membrane (CAM) model. See Fig. 4 on page 146.

\section{Photocopy Authorization Policy:}

Authorization to photocopy items for internal or personal use, or the internal or personal use of specific clients, is granted by Humana Press Inc., provided that the base fee of US $\$ 25.00$ per copy is paid directly to the Copyright Clearance Center at 222 Rosewood Drive, Danvers, MA 01923. For those organizations that have been granted a photocopy license from the CCC, a separate system of payment has been arranged and is acceptable to Humana Press Inc. The fee code for users of the Transactional Reporting Service is: [1-58829083-2/04 \$25.00].

Printed in the United States of America. $\begin{array}{lllllllllll}0 & 9 & 8 & 7 & 6 & 5 & 4 & 3 & 2 & 1\end{array}$

E-ISBN: 1-59259-658-4

Library of Congress Cataloging in Publication Data

Anticoagulants, antiplatelets, and thrombolytics / edited by Shaker A.

Mousa.

p. ; cm. -- (Methods in molecular medicine ; 93)

Includes bibliographical references and index.

ISBN 1-58829-083-2 (alk. paper)

1. Anticoagulants (Medicine) 2. Blood platelets. 3. Thrombolytic therapy. 4. Fibrinolytic agents.

[DNLM: 1. Anticoagulants--therapeutic use. 2. Thrombolytic

Therapy--methods. 3. Fibrinolytic Agents--therapeutic use. 4.

Heparin--therapeutic use. 5. Platelet Aggregation

Inhibitors--therapeutic use. 6. Thrombocytopenia--drug therapy. QZ 170

A629 2003] I. Mousa, Shaker A. II. Series.

RM340.A566 2003

615'.718--dc22 


\section{Preface}

During the past decade, remarkable progress has been made in the development of newer drugs to treat thrombotic and cardiovascular diseases. Over this last 10 years, concentrated efforts were made to discover new drugs and to develop novel uses for such traditional antithrombotic drugs as aspirin, heparin, and oral anticoagulants. Biotechnology, molecular biology, combinatorial approaches, isolation and characterization of natural products with antithrombotic activity, and a reassessment of traditional drugs have been the key factors behind these developments.

Some 1 million Americans die of thrombotic and cardiovascular disorders every year. On a global level, the relative proportion of mortality is even higher. Thus, thrombosis and related disorders represent the number one cause of mortality. The understanding of the mechanisms behind the pathogenesis of venous thromboembolism, acute coronary syndromes, cerebral vascular ischemic and thrombotic events, and diseases associated with thrombotic disorders has provided additional insights toward the development of various therapeutic approaches to control these pathogenic events. The roles of plasmatic proteins, blood cells, vascular endothelium, and target organs in both thrombogenesis, such as antithrombin III, Tissue Factor Pathway Inhibitor (TFPI), protein $\mathrm{C}$, prostacyclin, nitric oxide, and physiologic activators of fibrinolysis has led to the development of both direct and indirect modalities to treat thrombosis. On the other hand, the knowledge of the proteases involved in thrombogenesis, including tissue factors, coagulation factors, adhesion molecules, and fibrinolytic inhibitors have provided insights into the mechanisms by which thrombogenesis can be pharmacologically controlled.

The pharmaceutical industry has played a key role, not only in the development of new drugs, but also in providing sizable resources to academic institutions to foster the development and clinical validation of the uses of these drugs. Such drugs as low molecular weight heparins, antithrombin agents, the hirudins, such antiplatelet drugs as the GPIIb/IIa inhibitors, and the ADP receptor antagonists have emerged as improved therapeutic strategies over conventional drugs. The development of these drugs required a major undertaking by the pharmaceutical industry with allocation of sizable resources. Beside fiscal considerations, an objective assessment of the newer drugs at both the preclinical and clinical levels was mandatory for optimal applications.

Anticoagulants, Antiplatelets, and Thrombolytics provides a comprehensive update on many of the critical in vitro and in vivo models of thrombosis and hemostasis. Additionally, this book highlights the novel use of low molecular weight heparins, the newer applications of GPIIb/IIIa antagonists, aspirin, and clopidogrel, and the expanded usage of thrombolytics and polytherapeutic approaches. The recent approval of antiplatelet drugs for intermittent claudication, the aspirin-dipyridamol combination for ischemic stroke, and thrombolytic agents in cerebral ischemia represent some of the 
concepts that will be highlighted. Similarly, aspirin combinations with oral anticoagulants and other drugs have been in discussion for improved management of arterial thrombosis.

The recognition of heparin-induced thrombocytopenia as a catastrophic complication of heparin therapy necessitated the development of such alternate anticoagulant drugs as the antithrombin agents. Today, hirudins can be used to achieve comparable anticoagulation in heparin-compromised patients. The variation in aspirin tolerance and increased susceptibility to gastric toxicity from this drug led to the development of such ADP receptor antagonists as ticlopidine and clopidogrel. The oral anticoagulant drugs, however, have remained the only orally bioavailable agents that can be used for an extended period of time despite their inherent limitations. These drugs remain unchallenged by low molecular weight heparins, antithrombin agents, and antifactor Xa drugs. Thus, the impact of new drug development on traditionally used drugs has added a fresh dimension. It is interesting to note that many of the polytherapeutic approaches now combine traditional drugs with newer drugs.

The novel developments in antithrombotic drugs include such monotherapeutic approaches as the antiproteases (factors IIa, Xa, and VIIa), tissue factor targeting, platelet receptor targeting, and antithrombin III modulation. Despite the clear mechanisms of action of some of these agents, the recognition of the modulatory effects of aspirin and heparin has led to the development of a multitarget approach. Interestingly, all of the traditional drugs, such as heparin, aspirin, and warfarin, are multitargeting drugs. The recognition that thrombotic disorders represent a syndrome rather than a disease is of crucial importance in the development of newer drugs. Either a polytherapeutic approach with drug combinations or a drug with multiple actions will likely be more appropriate for the management of thrombotic disorders.

Shaker A. Mousa 


\section{Contents}

Preface

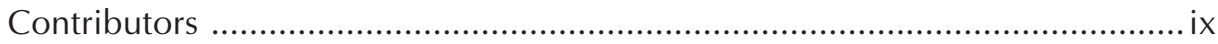

1 Highlights of Latest Advances in Antithrombotics

Shaker A. Mousa .................................................................................. 1

2 Antiplatelet, Anticoagulant, and Thrombolytic Drug Interactions

Omer Iqbal and Shaker A. Mousa

3 Evaluation of Platelet Antagonists in In Vitro Flow Models of Thrombosis

Owen J. T. McCarty, James P. Abulencia, Shaker A. Mousa, and Konstantinos Konstantopoulos

4 Heparin and Low Molecular Weight Heparin in Thrombosis, Cancer, and Inflammatory Diseases

Shaker A. Mousa

5 Are Low Molecular Weight Heparins the Same?

Shaker A. Mousa

6 Antithrombotic Drugs for the Treatment of Heparin-Induced Thrombocytopenia

Walter $P$. Jeske and Jeanine M. Walenga

7 Laboratory Methods for Heparin-Induced Thrombocytopenia

Margaret Prechel, Walter P. Jeske, and Jeanine M. Walenga

8 Factor Xa Inhibitors

Jeanine M. Walenga, Walter P. Jeske, Debra Hoppensteadt, and Jawed Fareed

9 Tissue Factor/VIla in Thrombosis and Cancer

Shaker A. Mousa

10 Tissue Factor Pathway Inhibitor in Thrombosis and Beyond

Shaker A. Mousa, Jawed Fareed, Omer Iqbal, and Brigitte Kaiser

11 Cell Adhesion Molecules: Potential Therapeutic and Diagnostic Implications

Shaker A. Mousa 
12 Development and Applications of Animal Models of Thrombosis

Ronald J. Shebuski, Larry R. Bush, Alison Gagnon, Liguo Chi, and Robert J. Leadley, Jr.

13 A Survey of Venous Thrombosis Models

Walter P. Jeske, Omer Iqbal, Jawed Fareed, and Brigitte Kaiser

14 Arixtra $^{\circledR}$ (Fondaparinux Sodium)

Shaker A. Mousa

15 Oral Thrombin Inhibitor Ximelagatran

Shaker A. Mousa

16 Pharmacogenomics and Coagulation Disorders

Omer Iqbal

17 Guidelines for Diagnosis and Treatment of Deep Venous Thrombosis and Pulmonary Embolism

Hikmat Abdel-Razeq, Mohamad Qari, Jorgen Kristensen, Hussein Alizeidah, Faisal Al-Sayegh, Mahmoud Marashi, Abdulaziz Alzeer, Omar Al-Amoudi, Hatem Qutub, Abdel-Aziz Al-Humiadi, Steen Husted, and Shaker A. Mousa on behalf of the GCC Thrombosis Study Group 


\section{Contributors}

Hikmat Abdel-RazeQ • King Fahd Armed Forces Hospital, Jeddah, Saudi Arabia James P. AbulEnCia - Department of Chemical and Biomolecular Engineering, Johns Hopkins University, Baltimore, MD

Omar Al-Amoud • King Abdul-Aziz University Hospital, Jeddah, Saudi Arabia Abdel-Aziz Al-Humiadi - King Faisal Specialist Hospital and Research Center, Jeddah, Saudi Arabia

Hussein Alizeidah • Tawam Hospital, Al Ain, United Arab Emirates

Abdulaziz Alzeer • King Khalid University Hospital, Riyadh, Saudi Arabia

FAISAl Al-SAYEGH • Health Sciences Center, Kuwait University, Kuwait

LARRY R. BUSH • Sepracor Inc., Marlborough, MA

Liguo CHI • Cardiovascular Pharmacology, Pfizer Global Research and Development, Ann Arbor Laboratories, Ann Arbor, MI

JAWED FAREED • Department of Pathology, Loyola University Medical Center,

Maywood, IL

Alison Gagnon • Incyte Genomics, Beverly, MA

Debra Hoppensteadt • Department of Pathology, Loyola University Medical Center, Maywood, IL

Steen Husted • Department of Cardiology, Aarhus University Hospital, Aarhus, Denmark

OMER IQBAL • Department of Pathology, Loyola University Medical Center, Maywood, IL

WAlter P. JeSKe • Departments of Thoracic-Cardiovascular Surgery and Pathology,

Cardiovascular Institute, Loyola University Medical Center, Maywood, IL

BRIGITTE KAISER • Center for Vascular Biology and Medicine Erfurt, Friedrich

Schiller University Jena, Erfurt, Germany

Konstantinos Konstantopoulos - Department of Chemical and Biomolecular

Engineering, Johns Hopkins University, Baltimore, MD

Jorgen Kristensen • Tawam Hospital, Al Ain, United Arab Emirates

Robert J. Leadley, JR. • Cardiovascular Pharmacology, Pfizer Global Research and Development, Ann Arbor Laboratories, Ann Arbor, MI

Mahmoud Marashi $\bullet$ Rashid Hospital, Dubai, United Arab Emirates

Owen J. T. MCCARTY - Department of Chemical and Biomolecular Engineering, Johns Hopkins University, Baltimore, MD

Shaker A. Mousa • Albany College of Pharmacy, Albany, NY

Margaret Prechel - Department of Pathology, Cardiovascular Institute, Loyola University Medical Center, Maywood, IL

Mohamad QARI • King Abdul-Aziz University Hospital, Jeddah, Saudi Arabia 
Hatem Qutuв • King Faisal University, Alkhobar, Saudi Arabia Ronald J. Shebuski $\bullet$ CarePoint Medical Inc., Eden Prairie, MN

Jeanine M. WaLenga - Departments of Thoracic-Cardiovascular Surgery and Pathology, Cardiovascular Institute, Loyola University Medical Center, Maywood, IL 